\title{
Nonlinear dual-core photonic crystal fiber couplers
}

\author{
José R. Salgueiro ${ }^{\dagger}$ and Yuri S. Kivshar \\ Nonlinear Physics Centre and Center for Ultra-high bandwidth Devices for Optical Systems (CUDOS), \\ Research School of Physical Sciences and Engineering, \\ The Australian National University, Canberra ACT 0200, Australia
}

\begin{abstract}
We study nonlinear modes of dual-core photonic crystal fiber couplers made of a material with the focusing Kerr nonlinearity. We find numerically the profiles of symmetric, antisymmetric, and asymmetric nonlinear modes, and analyze all-optical switching based on instability of the symmetric mode. We also describe elliptic spatial solitons controlled by the waveguide boundaries.
\end{abstract}

PACS numbers:

Photonic crystal fibers (PCF) have attracted a lot of attention due to their intriguing properties, potential applications, and the development of successful fabrication technologies [1]. PCFs are characterized by a conventional cylindric geometry with a two-dimensional lattice of air holes running parallel to the fiber axis. Such structures share many properties of photonic crystals, associated with the existence of the frequency gaps where the light transmission is suppressed due to Bragg scattering, as well as the guiding properties of conventional optical fibers, due to the presence of a core in the structure.

Recent theoretical and experimental results reported the studies and fabrication of dual-core PCF structures for broadband directional coupling or polarization splitting 2, 3, 4, 5, 6]. In PCFs, light confinement is restricted to the core of the fiber and therefore nonlinear effects, such as light self-trapping and localization in the form of spatial optical solitons [7], become important. In particular, similar to two-dimensional nonlinear photonic crystals [8], a PCF can support and stabilize both fundamental and vortex spatial optical solitons 9, 10. In a sharp contrast with an entirely homogeneous nonlinear Kerr medium where spatial solitons are unstable and may collapse, it was shown that the periodic structure of $\mathrm{PCF}$ can stabilize the otherwise unstable two-dimensional solitons.

In this Letter, we make a further step forward in the study of nonlinear effects in the PCF geometry, and analyze the existence and stability of nonlinear guided modes and spatial solitons in dual-core photonic crystal fiber couplers. The beam propagation and power-dependent switching in nonlinear directional couplers have been analyzed for the planar waveguide geometry 11]. Here we generalize those results for PCFs, as well as study the existence and stability of guided modes and elliptic spatial solitons controlled by the PCF holes. In particular, we find numerically the profiles of symmetric, antisymmetric, and asymmetric nonlinear modes, and analyze all-optical switching based on the mode instability.

We consider a simple model of PCF that describes, at a given frequency, the spatial distribution of light in a nonlinear dielectric material with a triangular lattice of air holes with the radius $r$ in a circular geometry. We assume that the PCF material possesses a nonlinear Kerr response, and there are two holes at the center filled by

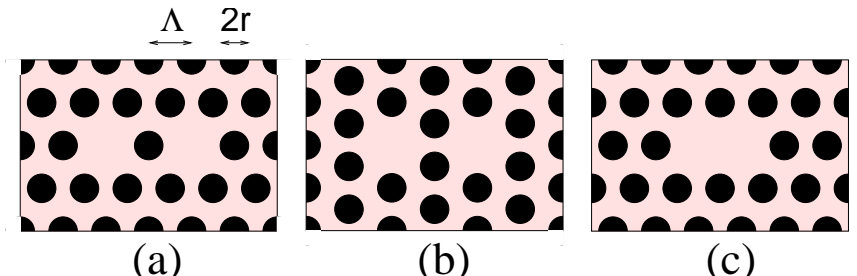

FIG. 1: (a-c) Schematic of three designs of a nonlinear dualcore photonic crystal fiber. The coupler is created by two missing neighboring holes in a nonlinear material.

the same material creating a nonlinear defect, as shown in Figs. 1(a-c). In the substrate material, the linear refractive index is $n_{s}$, whereas inside the holes it is $n_{a}$. In the nonlinear regime, the light distribution in PCF is described by the equation,

$$
-i \frac{\partial E}{\partial z}=\Delta_{\perp} E+W(x, y) E+V(x, y)|E|^{2} E,
$$

where $W(x, y)=n_{a}+\left(n_{s}-n_{a}\right) V(x, y), \Delta_{\perp}=\partial^{2} / \partial x^{2}+$ $\partial^{2} / \partial y^{2}$ is the transverse Laplacian, $E$ is the normalized electric field, and $V(x, y)$ is an effective potential describing two solid cores in the lattice of holes. We normalize $V=1$ in the material, and $V=0$ in the holes.

To find stationary nonlinear modes of PCF, we look for solutions in the form $E(x, y, z)=u(x, y) \exp (i \beta z)$, and obtain the nonlinear eigenvalue problem,

$$
\beta u=\Delta_{\perp} u+W(x, y) u+V(x, y)|u|^{2} u .
$$

In order to find the solutions of Eq. (2) for nonlinear localized modes, we consider a rectangular domain of the $(x, y)$ plane and apply a finite-difference scheme, taking respectively $N$ and $M$ uniformly distributed samples, $x_{i}, 0 \leq i<N$ and $y_{j}, 0 \leq j<M$ of the variables, as well as the corresponding samples for the stationary state, $u_{i j}=u\left(x_{i}, x_{j}\right)$ and the potential $V_{i j}=V\left(x_{i}, y_{j}\right)$. Substituting these variables into the model (2), and imposing homogeneous boundary conditions in all four edges of the domain, we obtain an algebraic nonlinear problem of $2 \times N \times M$ equations with the same number of unknowns $u_{i j}$, which is finally solved by means of a globally convergent Newton method. The presence of the external 
linear potential given by two missing holes and the lattice of air-holes makes the system non-scalable and its radial symmetry broken. Another approach, that takes an advantage of the lattice periodicity, was developed recently by Ferrando et al. [9].

First, we consider a single missing hole and find numerically solutions for the PCF spatial solitons [9]. Importantly, these stationary solutions are not perfectly radial, but they are stabilized by the PCF holes, in a sharp contrast with the unstable self-trapped beams in nonlinear focusing Kerr media. In order to demonstrate this feature, we follow the standard analysis of the soliton stability [7] and analyze the soliton power as a function of the soliton propagation constant. A positive slope of this dependence indicates the soliton stability.

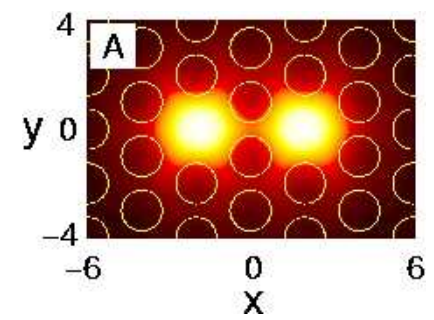

(a)

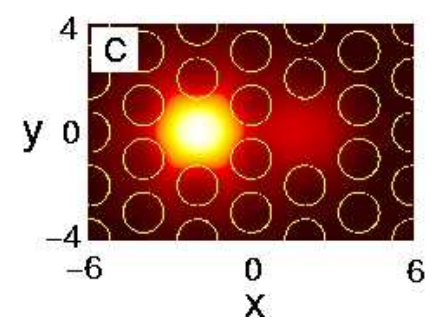

(c)

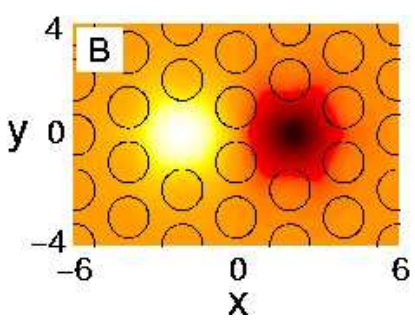

(b)

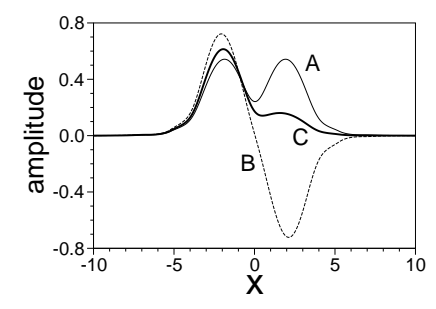

(d)
FIG. 2: (a-c) Light distribution between the two PCF cores in Fig. प(b) for three distinct nonlinear modes: symmetric (A), antisymmetric (B), and asymmetric (C). (d) Transverse profiles of the nonlinear modes $(\beta=3.95)$.

Next, we study the dual-core nonlinear PCFs shown in Figs. प(a,b), and find the families of the spatially localized modes-the so-called PCF spatial solitons-as a function of the mode propagation number $\beta$. The corresponding solutions are similar for two cases of Figs 1 $(a, b)$, and they can be envisaged as the modes of the effective dual-core fiber generated by the combined effect of the dual-core PCF refractive index and the nonlinear index induced by the mode amplitude itself. We find that the nonlinear dual-core PCF supports three distinct nonlinear modes, symmetric mode (A), antisymmetric mode (B), and asymmetric mode (C), as shown in Fig. 2 for the case of Fig. 1(b). The corresponding spatial profiles of these modes are shown in Fig. 2(d) as cross-section cuts along the line $y=0$. All the calculations are for $n_{s}=5, n_{a}=0, r=0.75$ and $\Lambda=2$.

To demonstrate the power relation between the modes, we follow the standard analysis of the soliton stability 12

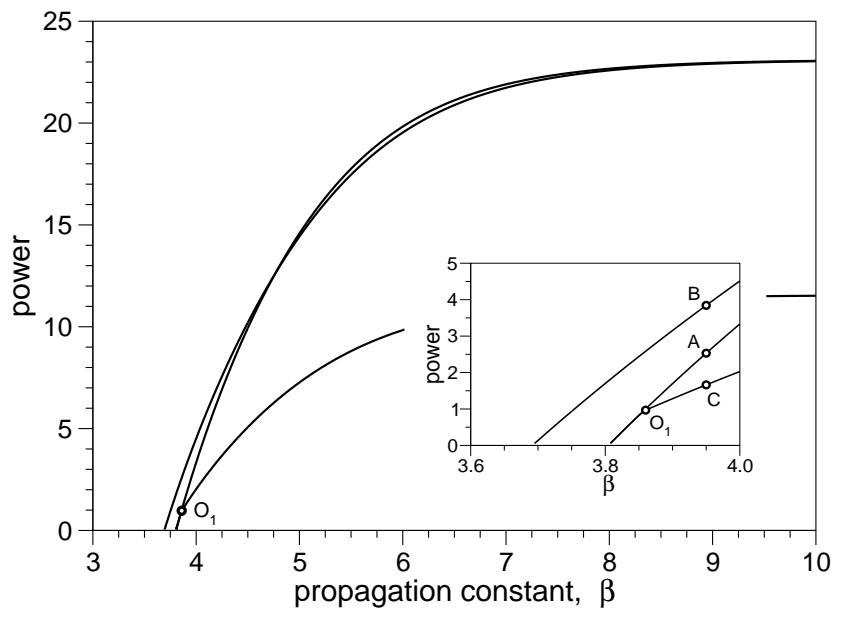

FIG. 3: Bifurcation diagram of the coupler modes. Insert shows an enlarged part near the bifurcation point. Asymmetric mode $\mathrm{C}$ bifurcates from symmetric mode $\mathrm{A}$ above a certain threshold in the mode power.

and plot in Fig. B the soliton power as a function of the soliton propagation constant, for the three different families. We notice that only two modes, symmetric and antisymmetric ones, may exist for low powers, whereas the asymmetric mode bifurcates from the symmetric mode at a ceratin threshold value of the mode power, above which the symmetric mode becomes unstable.

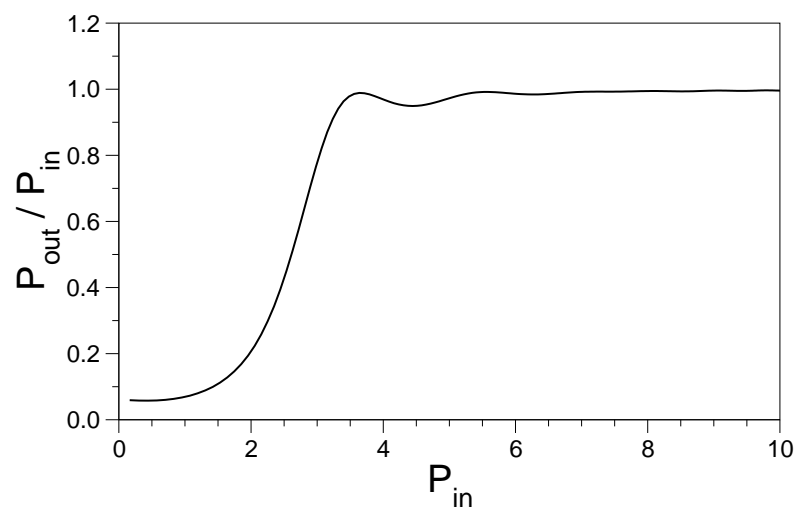

FIG. 4: Switching curve calculated for the PCF nonlinear coupler of Fig. 1(b). Due to instability of the symmetric mode, the light launched into one core only does not switch to the second core but remains in the same core.

In order to study the switching properties of the nonlinear coupler, we carry out a series of numerical simulations using the standard beam propagation method. First, we calculate the stationary mode of a singlecore PCF with the same parameters as the coupler corresponding to different powers. Then, this mode is launched into one of the cores of the dual-core coupler and, after some propagation, the output power is calculated at the same core. The propagation distance is determined considering the same coupler operating in the 

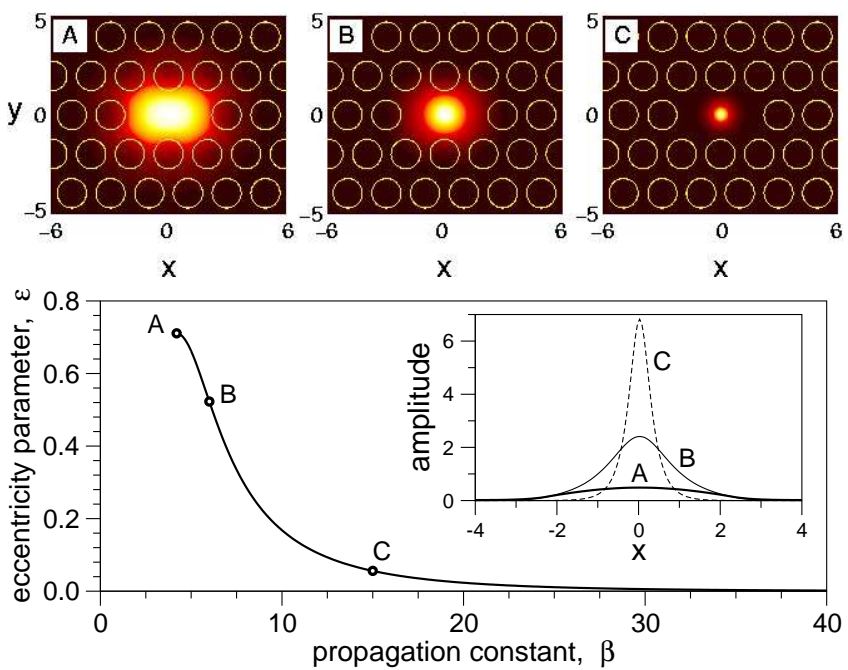

FIG. 5: Transformation of the elliptic guided modes to circular solitons for the growing mode power. Three examples on the top mark three points of the eccentricity curve for $\beta=4.2, \beta=6$, and $\beta=15$ respectively. Inset: the corresponding beam cross-sections at $y=0$.

linear regime and propagating the linear single-core mode launched to one of the coupler cores up to the point where all the energy is transferred to the second core. For the parameters used in our examples this point is reached at $z=21.23$. The switching curve is obtained by varying the input power for the fixed propagation length, and it

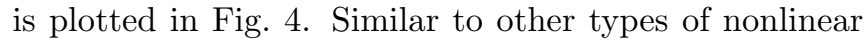
directional couplers, the input power is transferred completely to the second core for low powers, while for it remains in the initial one for higher input powers. A change between both the regimes takes place in a relatively short range of powers which constitutes a threshold where the power switching is triggered. For very low powers the energy is completely transferred to the second core, although from the plot in Fig. [ a residual amount seems to remain in the first one. This nonzero behavior of the curve close to the origin is explained by the overlapping of the field of the second core due to its proximity.

Finally, we study the third case of the closely spaced holes, shown in Fig. 1(c). In this case, there exists no bifurcation to the asymmetric state and the fundamental mode itself is elliptic as shown in the inset of Fig. 5 In the nonlinear case, this elliptic guided mode gives a birth to an elliptic spatial soliton controlled by the boundaries of the holes. In Fig. [5 we plot the mode eccentricity parameter $\epsilon=\left[1-\left(w_{x} / w_{y}\right)^{2}\right]^{1 / 2}$, where $w_{x}$ and $w_{y}$ are the mode axes (widths), and thus quantify the transformation of the elliptic guided modes (A) to the elliptic solitons (B) and then to the radially symmetric solitons (C). These results resemble the transformation of the shape of nonlinear guided modes in planar waveguides 13 .

In conclusion, we have demonstrated that several types of two-dimensional spatial optical solitons can be supported by a nonlinear dual-core PCF structure with the Kerr nonlinearity. We have analyzed numerically the existence and stability of symmetric, antisymmetric, and asymmetric nonlinear modes demonstrating that periodic refractive index of $\mathrm{PCF}$ provides also an effective stabilization mechanism for these composite localized modes to exist in a nonlinear Kerr medium, in a sharp contrast with an entirely homogeneous nonlinear Kerr medium where spatial solitons are known to be unstable, and they undergo the collapse instability. We have studied alloptical switching in the nonlinear dual-core PCF coupler associated with the instability of the symmetric mode.

The authors thank Adrian Ankiewicz for useful discussions and acknowledge a partial support of the Australian Research Council. JRS acknowledges a visiting fellowship granted by the Dirección Xeral de Investigación e Desenvolvemento of Xunta de Galicia (Spain), and he thanks Nonlinear Physics Center for a warm hospitality during his stay in Canberra.

${ }^{\dagger}$ On leave from: Facultade de Ciencias de Ourense, Universidade de Vigo, 32004 Ourense, Spain.
[1] Ph. Russell, Science 299, 358 (2003)

[2] F. Fogli, L. Saccomandi, P. Bassi, G. Bellanca, and S. Trillo, Opt. Express 10, 54 (2002).

[3] L. Zhang and Ch. Yang, Opt. Express 11, 1015 (2003).

[4] K. Saitoh, Y. Sato, and M. Koshiba, Opt. Express 11, 3188 (2003).

[5] H. Kim, J. Kim, U.-C. Paek, B.H. Lee, and K.T. Kim, Opt. Lett. 29, 1194 (2004).

[6] J. Lagsgaard, O. Bang, and A. Bjarklev, Opt. Lett. 29, 2473 (2004).

[7] Yu.S. Kivshar and G.P. Agrawal, Optical Solitons: From Fibers to Photonic Crystals (Academic, San Diego, 2003), $540 \mathrm{pp}$.
[8] S.F. Mingaleev, and Yu.S. Kivshar, Phys. Rev. Lett. 86, 5474 (2001).

[9] A. Ferrando, M. Zacarés, P. Fernandez de Córdoba, D. Binosi, and J.A. Monsoriu, Opt. Exp. 11, 452 (2003).

[10] A. Ferrando, M. Zacarés, P. Fernandez de Córdoba, D. Binosi, and J.A. Monsoriu, Opt. Exp. 12, 817 (2004).

[11] L. Thylen, E.M. Wright, G.I. Stegeman, C.T. Seaton, and J.V. Moloney, Opt. Lett. 11, 739 (1986).

[12] N.N. Akhmediev and A. Ankiewicz, Solitons (Chapman \& Hall, London, 1997).

[13] Q.Y. Li, C. Pask, and R.A. Sammut, Opt. Lett. 16, 1083 (1991). 